This report was prepared as an account of work sponsored by an agency of the United States Government. Neither the United States Government nor any agency thereof, nor any of their employees, makes any warranty, express or implied, or assumes any legal liability or responsibility for the accuracy, completeness, or usefulness of any information, apparatus, product, or process disclosed, or represents that its use would not infringe privately owned rights. Reference herein to any specific commercial product, process, or service by trade name, trademark, manufacturer, or otherwise does not necessarily constitute or imply its endorsement, recommendation, or favoring by the United States Government or any agency thereof. The vietvs and opinions of authors expressed herein do not necessarily state or reflect those of the United States Government or any agency thereof.
$\mathrm{L} E_{2}--10464-\mathrm{MS}$

DE85 016214

\title{
A Possibility for a Self-Consistent Treatment of Transport Processes in a Turbulent Plasma
}

J. P. Mondt 


\title{
A POSSIBILITY FOR A SELF-CONSISTENT TREATMENT \\ OF TRAYSPORT PROCESSES IN A TURBULENT PLASMA
}

by

$$
\text { J. P. Mandt }
$$

\section{ABSTRACT}

\begin{abstract}
Al1 commonly used models of plasma dynamics share a common flaw in their a priori validity. In particular, a solid foundation of plasma modelling on microscopic dynamics, as exists for moderately dilute gases, is obscured because of the difficulties inherent in the treatment of the potentially very important interplay between plasma waves and collisional processes. The present report briefly discusses the nature of these difficulties and presents $a$ possible approach towards the establishment of a plasma theory founded on the microscopic particle dynamics. The essence of this approach is the realization that only discrete particle interactions can create correlations. These therefore come into being on different spatial scales defending on their cluster number, after which collective effects magnify them analogous to the growth of initial perturbations in an unstable system. Truncation of the Bo-n-Bogolyubov-Green-Kirkwood-Yvon ('BBGKY') hierarchy thereby becomes a possibility through the introduction of a small parameter in inftial conditions although the dynainical system in itself does not contain a uniformly small parameter.
\end{abstract}

\section{INTRODUCTION}

The central problem in any transport theory is the determination of 1 ts correlation functions (1.e., functions describing the relation between the interaction among particles and their statistical distribution in phase 
space).1 For example, the spectral energy density, the free energy density and the collision Integral all are functionals of these correlation functions.

In the case of a turbulent plasma, there exists no satisfactory derivation of the size and functional form of correlation functions that is based on the fundamental equations of motion, the Klimontovich ${ }^{2}$ equation, or, In the case of a Hamiltonian system, the BBCKY hierarchy equations, 3 formally equivalent with Liouville's equation.

The present research proposition consists of an attempt to remedy this situation, thereby providing a microscopic foundation for the theory of transport processes in a turbulent plasma.

WEAK TURBULENCE

In the case of a weakly turbulent plasma (i.e., the energy density associated with the turbulent field fluctuations is much less than the thermal particle energy density), the shortcoming of the usual treatment consists in the neglect of particle-particle interactions in the derivation of the so-called "weak-turbulence kinetic equations." It is common practice to defend this neglect by referring to the smallness of the degree of collisionality, measurec by means of classical estimates for relaxation times and the range of the ensemble-averaged particle potential. But as is well-known, 5 these classical estimates do not hold in the presence of turbulent fluctuations. In fact, they cannot be trusted even in order of magnitude, since the presence of unstable roots of the dispersion equation, derived from a collisionless theory, translates into exponential growth and non-Markovian behavior of the collisien term that had been neglected from the outset.

Conclusively, what is needed in the theory of a weakly turbulent plasma is a self-consistent treatment of collisions and instabilities, i.e., a treatment that takes into account the effects of instabilities on collisional interactions before making any estimates of the effects of collisions on instabilities; thereby verifying whether the assumption of weak turbuler:e is preserved during any reasonable interval of time. 
In the case of a strongly turbulent plasma, transport theory so far has only been developed on the basis of model equations, not based on the fundamental equations of motion pertinent to a many particle system. ${ }^{6}$ This is due to the absence of any small parameter in the fundamental equations, except for the degree of collisionality that has beon put equal to zero from the outset.

However, only interactions can create correlations in a closed system. In passing it is noted that this circumstance is the reason for assuming vanishing initial correlations. ${ }^{7}$ Furthermore, of all the different types of interaction (wave-wave, particle-wave and particle-particle), only the particle-particle interactions constitute a true source of correlations. The only effect of the other types of interaction is to increase their amplitude in a way analogous to the increase in amplitude of a disturbance due to an Instability. 8

Consequentiy, the neglect of collisions in determining the size and functional form of correlations in a strongly turbulent plasma is not justifled no matter how small the degize of collisionality. Rather, a boundary layer analysis in the time variatie is neeied, such that, during the short time interval following the initial state, collisions are dominant. After this time Interval, collisional effects could tentatively be neglected, depending on the exact propertles of the system, and the correlation functions from then on are assumed to be subject mainly to wave-wave and wave-particle interactions. The mere fact that collisions constitute the source of correlations implies that their effect on them must exceed a11 other effects during $a$ short time interval in which such correlations are created. Not only do they have to be taken Into account: the fact that they are known to shape the different s-body correlations differently depending on the value of the cluster numbers, may very well imply sufficient discrimination between these various correlations to justify truncation of the BBGKY hierarchy. (Such a justification has recently been found for the case of a quiescent plasma. ${ }^{9}$ ) Thereby one could arrive at a kinetic description of the properties of a strongly turbulent plasma, despite the absence of any small parameter in the infinite set of hierarchy equations. 
OUTLINE OF THE REQUIRED RESEARCH PROGRAM

First it would have to be investigated under what conditions (if any) the effects of instabilities on collisions preclude a collisionless description of transport processes in a weakly turbulent plasma. If collisions could be consistently neglected, then the commonly adopted ${ }^{8}$ ordering of the different s-body irreducible correlations should be correct. Therefore, the three-body irreducible correlation could be neglected in the second hierarchy equation, resulting in a closed set of equations for the one-body distribution and the binary correlation. These equations could concelvably be solved as they were In the case of a quiescent plasma, using a newly developed perturbation approach $^{9}$. If the solution of these equations would show that collisions are important for the evolution of the one-body distribution function, then the first assumption made in the reasoning described above clearly must be incorrect. In that case, the relative orders of magnitude of the different correlation functions cannot be prescribed in an a priori manner; rather, they must be determined by the way they are built by collisions, as they were in the case of a quiescent plasma. ${ }^{9}$ In no way does one then still employ the assumption that the curb:lence is weak.

Conclusively, the first step in this research would elther: (1) Justify confidence in the assumption that collisional effects can be neglected on the evolution of correlation functions once these functions are significantly influenced by wave-wave and wave-particle interactions; or (2) Provide us with a drastically modified version of kinetic theory of a turbulent plasma, in which collisional effects have been properly taken into account.

In the first case, the boundary layer analysis of the evolution of correlations in the case of a strongly turbulent plasma could be simplified in a first attempt by neglecting such collisional effects on the evolucion of correlations after the initial small period of time during which collisional effects are necessarily dominating. As already mentioned, in the second case, the analysis of collisional effects on transport in a turbulent plasma does not depend in any essential way on the assumption of weak turbulence.

For simplicity, considerations will at first be restricted to electrostatic turbulence in an electron plasma, where the lons consticute a continuous, uniform background of positive charge. Appropriate generalization 
of the nonlinear hybrid-kinetic equations 10 can be contemplated as a straightforward method of describing fully electromagnetic turbulence.

\section{METHOD DF APPROACH}

Recently, an accurate approximation for the solution of the BBGKY hierarchy equations was obtained for the case of a quiescent plasma. $9^{9}$ The method was based on the observation that two widely distinct spatial scales exist in the system, one associated with strong collisions, the other with weak collisions. The absence of analytic behavior of the ove-body distribution function as a function of the plasma parameter, as well as tise impossibility of any spatially ,niform perturbation approach required construction of a new perturbation approach. Its success was mainly based on cercain scaling properties of operators in the BBGKY hierarchy equations. Since these properties are independent of the assumption of stability, there is sufficient reason to attempt a similar analysis for the case of a turbulent plasma.

\section{RELEVANCE}

As a rule, rather than as an exception, plasma is in a turbulent state. This holds for labcratory plasmas, such as present and thermonuclear generations of tokamaks, 11 reversed field pinches ${ }^{12}$ and stellarators ${ }^{13}$, as well as astrophysical plasmas, such as the solar wind.14 Without a microscopic foundation of the theory of plasma turbulence it will remain Impossible to make any quantitative predictions about this phenomenon, nor will tile-e be any true understanding of it within the framework of physics.

\section{REFERENCES}

1. N. N. Bogolyubov, "Problems of a Dynamical Theory in Stitistical Physics," State Technical Press (Moscow, 1946). Translated in "Studies in Statistical Mechanics," Vol. 1, Ed., J, de Boer and G. E. Uhlenbeck, North Holland Publ. Cy., (Amsterdam, 1962). 
2. Yu. L. Klimontovich, "The Statistical Theory of Non-equilibrium Processes in a Plasma," Pergamon Press, Ed., D. ter Haar (London, 1967).

3. M. Born and H. S. Green, "A General Kinetic Theory of Liquids," Cambridge University Press (London, 1949); J. G. Kirkwood, J. Chem. Phys. 14, 180 (1946) and 15, 72 (1947); J. Yvon, "La Theorle des Fluides et l'Equation d'Etat: Actualités Sclentifiques et Industrielles," Hermann et Cie. (Paris, 1935).

4. A. A. Vedenov, E. P. Velikhov and R. Z. Sagdeev, Nuclear Fuston 1, 82, (1961); Nuclear Fusion 2, 465 (1962).

5. D. C. Montgomery and D. A. Tidman, "Plasma Kinetic Theory," McGraw-H111, (New York, 1964).

6. B. B. Kadomtsev, "Plasma Turbulence," Academic Press (New York, 1965).

7. E. A. Frleman, J. Math. Phys. 4 , 410 (1963).

8. R. C. Davidson, "Methods in Nonlinear Plasma Theory," Academic Press (New York, 1972).

9. J. P. Mondt, Annals of Physics 117, 195 (1979).

10. D. A. D'Ippolito and R. C. Davidson, Phys. Flufds 18, 1507 (1975).

11. E. Mazzucato, Phys. Fluids, 21, 1063 (1978); Also see R. E. Slusher and C. M. Surko, Phys. Fluids 23, 472 (1980).

12. D. C. Robinson and M. G. Rushbrijge, Phys. Fluids 14, 2499 (1971).

13. K. Bol, Phys. Fluids $\underline{7}, 1855$ (1964).

14. M. L. Goldstein, R. A. Smith and K. Papadopoulos, The Astrophysical Journal 234, 683 (1979); Also see M. V. Goldman, G. F. Relter and D. R. Nicholson, Phys. Fluids 23, 388 (1980). 